Service social

\title{
Les transformations dans les services à domicile en Ontario
}

\section{Maurice Aumond et Francine Beaulieu}

Volume 43, numéro 1, 1994

Maintien à domicile

URI : https://id.erudit.org/iderudit/706646ar

DOI : https://doi.org/10.7202/706646ar

Aller au sommaire du numéro

Éditeur(s)

École de service social de l'Université Laval

ISSN

1708-1734 (numérique)

Découvrir la revue

Citer cet article

Aumond, M. \& Beaulieu, F. (1994). Les transformations dans les services à domicile en Ontario. Service social, 43(1), 127-142.

https://doi.org/10.7202/706646ar

\section{Résumé de l'article}

L'organisation des services de soins de longue durée en Ontario est fort complexe. Ces services font actuellement l'objet d'une réévaluation en profondeur. Les auteurs de l'article présentent d'abord l'organisation actuelle des services de soins de longue durée. Puis, dans un deuxième temps, à la lumière de la situation présentée, ils discutent des effets de la fragmentation des services. En troisième analyse, ils mettent en évidence les mesures proposées à travers les nouvelles politiques gouvernementales en ce qui a trait aux services à domicile et, par la suite, se penchent sur l'efficacité des transformations proposées. En guise de conclusion, ils mettent en relief quelques aspects positifs des changements suggérés et dégagent quelques appréhensions majeures face à la réforme en cours, particulièrement en ce qui a trait aux services à domicile. 


\title{
Les transformations dans les services à domicile en 0 ntario
}

\author{
Maurice AUMOND \\ Ph.D., Professeur-chercheur \\ École de service social, Université Laurentienne \\ Sudbury, Ontario \\ Francine BEAULIEU \\ Chargée d'enseignement \\ École de service social, Université Laurentienne \\ Sudbury, Ontario
}

Le présent article s'adresse d'abord aux professionnelles et aux professionnels qui travaillent en maintien à domicile dans les régions frontalières du Québec avec I'Ontario, mais aussi à celles et à ceux qui se préoccupent des soins de longue durée dans cette province. Il est destiné à susciter et à alimenter des discussions tant sur l'organisation des services que sur le rôle et l'impact des divers programmes auprès de la clientèle.

Les soins de longue durée en Ontario subissent actuellement de profondes transformations, tant sous l'aspect de la planification et de I'administration que sur le plan des modes de distribution des services. À cet égard, en octobre 1991 la province entreprenait une vaste 
consultation à propos de l'actuelle direction du gouvernement dans les soins de longue durée (Ontario, 1991a).

À la suite de cette tournée, le gouvernement social-démocrate de l'Ontario, alors nouvellement élu, a entrepris une vaste réforme visant à amener les ministères, les organismes engagés dans les soins de longue durée ainsi que les utilisatrices et les utilisateurs de services à travailler en partenariat. Cette nouvelle stratégie voulait faire en sorte que les services puissent répondre à une clientèle élargie. Elle visait également à diminuer la fragmentation et le dédoublement des services et à réduire la dépendance de cette même clientèle à l'égard des établissements (Ontario, 1991a).

Afin de bien saisir la portée de ces mesures, nous nous intéresserons particulièrement à la fragmentation des services dans les soins de longue durée. Pour ce faire, nous dresserons d'abord un portrait de l'organisation actuelle des services. Ensuite, à la lumière de la situation présentée, nous discuterons des effets de la fragmentation des services. Nous terminerons en mettant en évidence les mesures proposées en ce qui à trait aux services à domicile, puis en analysant l'efficacité de l'ensemble des mesures mises en avant par cette réforme.

\section{PORTRAIT DE L'ORGANISATION GÉNÉRALE DES SERVICES}

L'intérêt de notre démarche réside dans le fait que peu d'auteurs se sont penchés sur la portée de la nouvelle politique de soins de longue durée en Ontario. Ainsi, ces mesures étant en implantation, elles n'ont pas encore fait l'objet d'évaluation. Par le fait même, il n'existe guère de documentation à ce sujet, tant en français qu'en anglais (Beaulieu, 1994). Néanmoins, durant la dernière décennie, plusieurs études gouvernementales ont dénoncé la fragmentation des services.

À cet égard, signalons les documents suivants: Nouvelles orientations. Services de santé et services sociaux à l'intention des personnes âgées de l'Ontario (1986), rédigé à l'intention du gouvernement libéral provincial de l'époque, et Nouvelle orientation en matière de soins de longue durée et de services de soutien en Ontario (1991a), élaboré par le gouvernement provincial néo-démocrate actuel. Ces documents présentent les grandes orientations ministérielles en matière de soins de longue durée adoptées respectivement par les deux partis politiques qui se sont succédé à Queen's Park.

À ces documents gouvernementaux s'ajoutent les études de la firme Price Waterhouse. En effet, à la demande du ministère de la Santé, celle-ci a élaboré une Étude opérationnelle du programme de soins à domicile de l'Ontario: rapport final (1989). Le même groupe a procédé 
à l'Évaluation des services intégrés d'aides familiales: rapport final (1989), mais, cette fois, commanditée par le ministère des Services sociaux et communautaires. D'un autre côté, Evans et Book (1990) ont rédigé un Rapport du Comité d'étude provincial-municipal sur les services sociaux. Finalement, une dernière étude, Des racines rurales: Le vieillissement dans les petites localités et les régions rurales de l'Ontario (1992), a été réalisée par le Conseil conultatif de l'Ontario sur l'âge d'or.

Ces études couvrent de façon particulière certains volets des services à domicile, dont les soins à domicile, les services des aides familiales ainsi que les services de soutien communautaire. Elles mettent en lumière la nécessité d'étendre le réseau de services de soins de longue durée, de façon que les personnes âgées aient accès à un minimum de services partout en Ontario. Ces rapports de recherche recommandent d'assurer une coordination plus efficace des programmes entre les différents ministères engagés dans les soins de longue durée ainsi qu'à l'intérieur de ces ministères. Bref, avec l'adoption de cette nouvelle politique, les partenaires engagés dans les soins de longue durée en Ontario souhaitent que les difficultés qui ont été reconnues par les études mentionnées plus haut soient résolues.

Afin de mieux saisir la portée de la nouvelle politique, voici le portrait de la situation actuelle. Tout d'abord, signalons qu'en Ontario les services à domicile sont fournis soit par des organismes communautaires, soit par des organismes parapublics et que, de ce fait, les règles du jeu ne sont plus les mêmes; ainsi, les critères d'admission sont différents, les territoires desservis ne sont pas les mêmes et les modes de financement sont très diversifiés. À cause de ces diversités il devient difficile de rendre les services complémentaires, en particulier dans le champ des services à domicile. Cette situation entraîne même de la compétition entre les organismes et pénalise finalement la clientèle, puisque celle-ci ne peut recevoir des services en provenance de deux programmes en même temps. Qui plus est, la multiplication des acteurs et des organismes à l'intérieur d'un même programme rend difficile I'accès aux services et augmente les coûts de gestion.

Les soins de longue durée comprennent donc toute une panoplie de programmes et de services, notamment les services communautaires de soutien, les services de santé et de soutien personnel, le logement offrant des services de soutien, les services de coordination des placements, les établissements de soins de longue durée, les hôpitaux pour malades chroniques et les lits pour malades chroniques dans les hôpitaux de soins actifs, les maisons de repos et de retraite et les programmes d'avantages sociaux (Ontario, 1993b: 11-15).

Contrairement à ce qui existe au Québec, les soins de longue durée en Ontario englobent la gamme complète des services offerts 
aux personnes âgées en perte d'autonomie, y compris les services de maintien à domicile, les services d'hébergement en centre $d^{\prime}$ accueil ou dans des centres de soins prolongés. À titre indicatif de ce qui existe dans les grandes villes de la province, nous produisons en annexe la nomenclature de ces programmes et services à partir de ceux existant pour la Municipalité régionale de Sudbury. Cependant, notre analyse de la politique des soins de longue durée porte uniquement sur le volet des services à domicile.

Le volet des services à domicile contenu dans la politique des soins de longue durée s'articule autour de deux programmes distincts: le Programme de services de santé et de soutien personnel et le Programme de services de soutien communautaire.

Le Programme des services de santé et de soutien personnel comprend les services suivants :

- les soins infirmiers,

- I'ergothérapie,

- la physiothérapie,

- I'orthophonie,

- le travail social,

- la consultation nutritionnelle,

- les soins personnels, les services de soins auxiliaires et les travaux ménagers essentiels,

- le soutien des soignants (information, instructions et soins de relève),

- certains produits et services (ex. : les médicaments, les fournitures et les équipements médicaux) dans les circonstances prévues (Ontario, 1993b: 33-34).

Quant au Programme de services de soutien communautaire, il fournit les services tels que :

- les services des repas (Meals on Wheels et Diners Club),

- le transport,

- I'entretien du foyer,

- les visites amicales,

- les visites de surveillance, d'information et d'aiguillage,

- les programmes de jour pour adultes,

- les Centres pour personnes âgées,

- des services spécialisés, comme les programmes communautaires pour la maladie d'Alzheimer (Ontario, 1993c: 28).

Comme nous pouvons le constater, les nombreux services sont très fragmentés, contrairement à ce qui se passe au Québec où les services sociaux et de santé sont regroupés sous un même ministère, 
celui de la Santé et des Services sociaux. C'est ce même ministère qui finance le réseau des affaires sociales et de la santé. À l'inverse, en Ontario, les services sont subventionnés par plus d'un ministère, en I'occurrence celui de la Santé, ceux des Services sociaux et communautaires, de l'Éducation et de la Formation ainsi que du Logement. De surcroît, les organismes fournisseurs de services relèvent de paliers paragouvernementaux, provinciaux, municipaux, bénévoles et privés (Evans et Book, 1990: 199).

À la lecture de ce qui précède, nous constatons qu'une variété d'organismes offrent une gamme de services à la population âgée tant à domicile qu'en milieu d'hébergement. Force nous est alors de constater aussi que le type de gestion des agences, leurs modes de financement ainsi que la liste de leurs services illustrent bien le dédoublement des services, de même que la complexité et la diversité dans les sources de financement.

Aussi la lectrice ou le lecteur devront-ils être attentifs, puisque le vocabulaire utilisé ici diffère de celui employé au Québec, particulièrement dans le réseau de la santé et des services sociaux.

\section{LES EFFETS DE LA FRAGMENTATION DES SERVICES}

Le ministère de la Santé ainsi que le ministère des Services sociaux et communautaires reconnaissent que les services de soins de longue durée, plus particulièrement les services à domicile, sont fragmentés. De l'aveu même du gouvernement,

un des défauts actuels des soins de longue durée et des services de soutien est qu'ils sont fragmentés ou qu'ils ne sont tout simplement pas disponibles. Des services similaires sont offerts en vertu de différents programmes mais ont des critères $\mathrm{d}^{\prime}$ admission, des modes de financement et des normes de services différents. Les gens trouvent cette situation très déroutante et ils peuvent avoir de la difficulté à trouver l'aide dont ils ont besoin (Ontario, 1991a: 3).

Cela étant dit, regardons comment se vit la fragmentation dans les services. Cette fragmentation s'explique par le fait que ces services ne sont pas disponibles partout en Ontario et que leur accès est limité. Les difficultés associées à l'accessibilité sont aussi attribuables à la similitude entre les programmes, aux divers critères d'admissibilité, aux contributions exigées des utilisatrices et des utilisateurs des services dans certains cas et à l'absence de services dans certaines régions (Ontario, 1991a: 3). Leur accessibilité et leur disponibilité deviennent encore plus restreintes pour la clientèle francophone. 
Quant à l'accessibilité des services à domicile, ceux-ci n'étant pas disponibles partout en Ontario, il faut en élargir la gamme. Parmi les services à mettre en place à la grandeur de la province, mentionnons les services d'aides familiales, les services de coordination de placement et les services communautaires de soutien (Ontario, 1991a: 18; Ontario, 1991b: 57 ; Ontario, 1992: 2).

Les sources de financement constituent, à notre avis, une autre façon de restreindre l'accessibilité aux services. Les agences sollicitent auprès de plusieurs instances gouvernementales des subventions dans le but de combler un besoin particulier dans la communauté. Elles reçoivent ainsi leur financement de sources diverses. Bien entendu, les priorités des organismes subventionnaires diffèrent selon les ministères de sorte que les critères d'admissibilité deviennent difficilement complémentaires entre les agences.

Dans un tel contexte, les utilisatrices et les utilisateurs peuvent subir des interruptions de services, le temps d'être admis dans un autre programme. Dès lors, l'attribution des budgets ne tient pas compte de l'ensemble des besoins de la clientèle, mais bien d'une partie seulement de leurs besoins.

Les sommes exigées pour obtenir certains services contribuent également à cette fragmentation. En changeant d'organisme, il est possible que la personne âgée doive débourser de l'argent pour obtenir le même type d'aide qu'elle recevait gratuitement auparavant (Ontario, 1991a: 43). Dès lors, elle s'explique mal, et à juste titre, pourquoi tel service est gratuit avec un certain organisme, alors qu'elle doit en payer une partie avec tel autre.

La segmentation s'explique aussi par le fait que plusieurs programmes offrent des services similaires (Ontario, 1991a: 3). Ce phénomène nous apparaît plus évident dans l'ensemble des services à domicile. En fait, ce volet des soins de longue durée compte quatre programmes analogues:

- le Programme de soins à domicile,

- le Programme intégré de services d'aides familiales,

- le Programme de services d'auxiliaires à domicile et

- le Programme d'aides familiales et d'infirmières visiteuses

(Ontario, 1993b : 12).

En revanche, le fait que les services se ressemblent d'un programme à l'autre, mais sans être nécessairement complémentaires, permet une plus grande flexibilité sur le plan administratif. Ainsi, un organisme qui ne possède pas de personnel dans une localité ou une région qu'il doit desservir peut acheter, au besoin, les services d'un autre programme offrant des services similaires. En contrepartie, cette 
manœuvre administrative peut contribuer à semer la confusion chez les utilisatrices et les utilisateurs de services, parce que l'intervenante ou l'intervenant relève d'un organisme autre que celui auquel ils ont fait appel.

De surcroît, la recherche de Beaulieu (1994) confirme que la confusion peut être encore plus grande lorsqu'une contribution financière est demandée simplement parce que la condition de santé de I'utilisatrice ou de l'utilisateur s'est modifiée sensiblement. Ainsi, lorsque l'état de santé ne nécessite plus de soins infirmiers et que l'utilisatrice ou l'utilisateur continue d'avoir besoin d'une ou d'un auxiliaire à domicile, on l'oriente alors vers un nouveau service qui exige une contribution financière. En sollicitant ce service dans cette circonstance, la personne reçoit la visite de la même intervenante ou du même intervenant qui effectuera les mêmes tâches qu'auparavant, mais à la condition que la personne bénéficiaire verse une contribution cette fois-ci... Ainsi, la personne âgée éprouve de la difficulté à comprendre que le service qui était gratuit, parce qu'il était relié à un problème particulier de santé, ne l'est plus et que, dorénavant, elle doit en payer les coûts.

Comme nous l'avons mentionné précédemment, le financement et la gestion des programmes proviennent de sources différentes. La distribution des services s'opère à partir de critères $\mathrm{d}^{\prime} \mathrm{admission}$ propres à chaque programme, sans qu'il y ait de concertation. De surcroît, les fonctions et les responsabilités en matière de prestation et de financement des services sociaux n'ont pas été révisées depuis qu'elles ont été établies au cours des années 60 (Evans et Book, 1990). Pourtant, ces programmes avaient été mis en place pour se compléter mutuellement et ainsi pouvoir offrir à la clientèle des services continus.

Pour contrer cette difficulté, Evans et Book (1990) recommandent de favoriser l'établissement de liens entre les services sociaux et les autres processus de planification dans le milieu. Une planification communautaire pourrait contribuer à améliorer la collaboration et la coordination entre les réseaux de services et à réduire le fractionnement des services et éliminer ceux qui se chevauchent.

En conséquence, la fragmentation des services engendre plusieurs difficultés pour les personnes âgées. Elle compromet la continuité des soins, l'accessibilité aux services et, par le fait même, oblige les personnes âgées à marchander parmi la gamme des services offerts. Cette situation entraîne un sentiment d'insécurité parmi la clientèle. Elle favorise la multiplicité des organismes de services ainsi que des intervenantes et des intervenants auprès des utilisatrices et des utilisateurs, portant ainsi, selon nous, atteinte à leur droit d'accès à des services 
appropriés. Finalement, la fragmentation des services contribue à maintenir une vision parcellaire des besoins des personnes âgées (Beaulieu, 1994).

\section{LES MESURES PRO PO SÉES ET LEUR EFFICACITÉ}

Le ministère de la Santé ainsi que le ministère des Services sociaux et communautaires manifestent, depuis I'adoption de la nouvelle politique déjà mentionnée, leur volonté de réduire la fragmentation dans les services de soins de longue durée, afin de faciliter l'accessibilité, de diminuer le dédoublement des services et de redistribuer les ressources.

Les mesures annoncées dans cette nouvelle politique sont en voie d'implantation dans la province. Parmi les modifications prévues, celles reliées aux services à domicile sont:

- l'intégration de la gestion des soins de longue durée provenant des ministères de la Santé et des Services sociaux et communautaires;

- la formation de comités locaux de planification par les Conseils régionaux de santé;

- I'extension des programmes intégrés d'aides familiales à toutes les régions de la province;

- la fusion des programmes des soins à domicile aux malades aigus et aux malades chroniques et du Programme intégré d'aides familiales dans le cadre du nouveau Programme de services de santé et de soutien personnel;

- la refonte de la gamme des services communautaires en un seul programme, le Programme de services de soutien communautaire;

- l'élargissement des soins palliatifs;

- la création d'organismes de services polyvalents (Ontario, 1991a; Ontario, 1993c).

La plupart de ces mesures sont déjà mises en application ou sont en voie de réalisation, à l'exception de la création des organismes de services polyvalents (OSP).

Il y a lieu, sans doute, de se demander si les mesures proposées parviendront à réduire la fragmentation. Pour ce faire, nous avons utilisé la grille d'évaluation de l'implantation des services de santé mentale. Cet instrument de mesure élaboré par Pelletier et Boyer (1988) permet d'évaluer plusieurs programmes, dont des programmes ou services de maintien, de soutien, de réadaptation et d'accompagnement dans la communauté. Les critères d'évaluation portent sur l'accessibilité et la disponibilité, la coordination, la continuité, la personnalisation, la 
communautarisation et la qualité de vie (Pelletier et Boyer, 1988: 4-5). Vous en trouverez les définitions à l'annexe II.

Les indicateurs d'évaluation pour mesurer l'accessibilité des services sont la variété et la flexibilité des moyens pour répondre aux besoins spécifiques de la clientèle, l'information du milieu et de la population cible, la facilité d'accès et la disponibilité des ressources.

Les moyens mis en œuvre pour répondre aux besoins de la clientèle âgée à domicile nous apparaissent variés et flexibles. En effet, il existe deux programmes destinés à répondre aux besoins de la clientèle à domicile, soit le Programme de services de santé et de soutien personnel et le Programme de services de soutien communautaire. La clientèle accède aux services de l'un ou l'autre de ces programmes en fonction de son état de santé. Cependant, les personnes âgées connaissent peu les services à domicile (Beaulieu, 1994; Sudbury, 1991). Les travaux de Beaulieu (1994) montrent que les personnes âgées ont appris l'existence des services au moment où elles en ont eu besoin seulement, c'est-à-dire au moment de leur hospitalisation ou à l'occasion d'une visite chez leur médecin.

Les difficultés d'accès aux services ont été dénoncées dans plusieurs études, notamment celles du gouvernement de l'Ontario (1986 et 1991a), d'Evans et Book (1990) et du Conseil consultatif de l'Ontario sur l'âge d'or (1992). Pour contrer cette difficulté, deux mesures ont été prises.

La première consiste à regrouper plusieurs services en un seul programme, comme c'est le cas pour le Programme de services de santé et de soutien personnel et pour le Programme de services de soutien communautaire. En procédant ainsi, les critères d'admissibilité sont unifiés à l'intérieur de chacun de ces programmes. Cette mesure rend plus uniforme la prestation des services d'une région à l'autre. Toutefois, I'unification des services à domicile en deux programmes ne signifie pas la fusion des organismes qui dispensent ces services (Ontario, 1991a: 23-26) et ne garantit pas nécessairement plus de continuité dans les services offerts à la clientèle. Beaulieu (1994) constate l'absence de liens fonctionnels et de communication entre les deux programmes. Les besoins des personnes âgées y sont évalués en fonction des réalités d'un service et non en fonction d'un réseau de services. De surcroît, la clientèle ne peut recevoir des services de deux programmes en même temps.

De notre point de vue, cette mesure facilite l'accès aux services à l'intérieur de chacun de ces programmes. Elle continue à maintenir la multiplicité des organismes et des intervenantes et intervenants auprès de la clientèle et elle perpétue une vision parcellaire des besoins des utilisatrices et des utilisateurs. 
Le deuxième moyen privilégié pour améliorer l'accessibilité des services consiste en la création d'organismes de services polyvalents (OSP). Ces nouveaux organismes seront développés à partir de la fusion des organismes qui assurent actuellement une partie ou la totalité des soins de longue durée (Ontario, 1993d). Leur mandat sera d'offrir et de coordonner les services provenant du Programme de services de santé et de soutien personnel et du Programme de services de soutien communautaire. Plus spécifiquement, les OSP seront responsables:

- d'offrir un accès simplifié à un éventail de soins de longue durée et de soutien...;

- d'offrir aussi des services de renseignements et d'orientation pour les services de soins de longue durée et les autres services communautaires;

- de constituer un point central pour la coordination et l'intégration des services;

- de diriger et d'offrir directement les services communautaires de soins de longue durée (Ontario, 1993d: 13).

Leur rôle permettra l'accès unifié par une seule porte d'entrée aux services essentiels de soins de longue durée et de soutien. Les OSP proposeront un service de gestion de cas afin de déterminer l'admissibilité au soutien communautaire et aux soins de longue durée (y compris les admissions dans les établissements de soins de longue durée et l'orientation vers les programmes de gériatrie). De plus, les OSP veilleront à ce que les usagers obtiennent les services dont ils ont besoin et ils surveilleront leur évolution. Finalement, ils s'assureront que ceuxci tiennent compte des caractéristiques géographiques, linguistiques et culturelles des usagers (Ontario, 1993d). Le développement des OSP facilitera donc l'accessibilité, puisque les usagers s'adresseront désormais à un seul endroit pour obtenir les services dont ils ont besoin. De plus, la fonction de gestion de cas permettra de coordonner les services et de réévaluer les besoins changeants de la clientèle.

Les OSP représentent la pierre angulaire de la réforme des soins de longue durée en Ontario. Ces nouveaux organismes suscitent l'espoir ultime que prenne fin la fragmentation dans les soins de longue durée et que soit facilitée l'accessibilité aux services. Ils constitueront le point névralgique pour la coordination et l'intégration des services.

En ce qui a trait à la disponibilité des ressources, l'extension des programmes intégrés d'aides familiales et des soins palliatifs à la grandeur de l'Ontario rendra ces services disponibles pour la population, eu égard à son lieu de résidence (Ontario, 1993a). 
Somme toute, les mesures proposées visent dans l'ensemble à améliorer l'accessibilité et la disponibilité des ressources. Elles supposent l'utilisation plus efficace des ressources, une réponse plus adéquate à la clientèle et une diminution des dédoublements.

Pour éliminer les chevauchements de services et pour améliorer la coordination, la gestion des soins de longue durée provenant des ministères de la Santé et des Services sociaux et communautaires a été intégrée. De plus, des comités locaux de planification ont été formés par les Conseils régionaux de santé.

Pour plus d'efficacité, des bureaux locaux de soins de longue durée ont été ouverts dans les régions administratives de la province. Leur rôle consiste à établir un réseau complet et coordonné de soins de longue durée et de services de soutien, à affecter les ressources budgétaires dans leurs régions respectives et à évaluer l'efficacité des services offerts (Ontario, 1991a: 55-56).

Les comités locaux de planification, mis sur pied par les Conseils régionaux de santé, ont la responsabilité d'élaborer un plan de soins de longue durée, de formuler les recommandations quant au financement local des services, d'aider les communautés à planifier les OSP et de s'assurer que ceux-ci répondent aux besoins locaux (Ontario, 1993d: 29).

L'intégration de la gestion des services de santé et sociaux et la mise sur pied des comités locaux de planification visent donc à permettre une meilleure coordination et à assurer la continuité de services régionaux et locaux dans les soins de longue durée.

L'expérience ontarienne en matière d'organisation de services à domicile nous montre l'importance d'acquérir une vision globale de la prestation des services à une clientèle et $d^{\prime}$ articuler cette vision à I'intérieur d'un même programme. L'unification des programmes permettrait l'harmonisation des critères d'admission et des normes de services et rendrait plus facile la complémentarité entre les divers services fournis à partir d'un programme unique.

Notre analyse expose également les difficultés engendrées par la multiplication des organismes.

\section{CONCLUSION}

L'élaboration de la nouvelle politique en matière de soins de longue durée en Ontario a permis de dresser un portrait réel de la complexité de l'organisation des services et de définir quelques problèmes sousjacents. 
Comme nous l'avons fait valoir plus haut, la fragmentation compromet l'accessibilité aux services et la continuité des soins. Elle favorise la multiplicité des organismes de services et des personnes intervenant auprès de la clientèle. De surcrôit, elle contribue à maintenir une vision parcellaire des besoins des personnes âgées.

Les mesures qui ont été mises en œuvre jusqu'à maintenant nous apparaissent insuffisantes pour éliminer la fragmentation. Ainsi que nous l'avons vu, les mesures proposées visent à améliorer la coordination locale et régionale et à étendre à la grandeur de la province les services de santé, de soutien personnel et de soutien communautaire.

Le développement des organismes de services polyvalents (OSP) demeure l'espoir ultime pour les partisans de la nouvelle politique des soins de longue durée de voir enrayer la fragmentation dans les soins de longue durée. Ce nouvel organisme qu'est l'OSP sera-t-il en mesure $d^{\prime}$ imposer sa philosophie de gestion et $d^{\prime}$ 'intervention auprès des agences et du personnel engagés dans les soins de longue durée depuis nombre d'années?

\section{Références bibliographiques}

BeAUlieu, F. (1994). L'accessibilité des services à domicile pour les personnes âgées francophones de la Municipalité régionale de Sudbury. Sudbury: Université Laurentienne, École de service social, mémoire de maîtrise. Inédit.

CONSEIL CONSULTATIF DE L'ONTARIO SUR L'ÂGE D'OR (1992). Des racines rurales. Le vieillissement dans les petites localités et les régions rurales de l'Ontario. Toronto.

Evans, C. et R. BOOK (1990). Rapport du Comité d'étude provincial-municipal sur les services sociaux. Toronto: Imprimeur de la Reine pour I'Ontario.

home Care Program (1993). Le Programme de soins à domicile. Sudbury. Dépliant préparé par le Service de santé publique.

ONTARIO (1993a). L'expansion des services d'aide familiale et la nouvelle politique de soins palliatifs doivent améliorer les services de soins de longue durée. Toronto : ministère de la Santé. Communiqué nº 92 /nr-156.

ONTARIO (1993b). Les partenariats dans les soins de longue durée. Cadre d'élaboration des politiques. Toronto: Imprimeur de la Reine pour I'Ontario.

ONTARIO (1993c). Les partenariats dans les soins de longue durée. Cadre de mise en œuvre. Toronto: Imprimeur de la Reine pour l'Ontario.

ONTARIO (1993d). Les partenariats dans les soins de longue durée. Directives d'établissement des organismes de services polyvalents. Toronto: Imprimeur de la Reine pour l'Ontario. 
ONTARIO (1992). Objet: soins de longue durée. Toronto: allocution de la ministre de la Santé, Frances Lankin, prononcée devant I'Assemblée législative (2 décembre).

ONTARIO (1991a). Nouvelle orientation en matière de soins de longue durée et de services de soutien en Ontario (document de consultation). Toronto: Imprimeur de la reine.

ONTARIO (1991b). Guide à l'intention des personnes âgées. Toronto: Imprimeur de la Reine pour I'Ontario.

ONTARIO (1991c). Comment choisir le bon endroit pour vivre. Toronto: Imprimeur de la Reine pour l'Ontario.

ONTARIO (1986). Nouvelles orientations. Services de santé et services sociaux à l'intention des personnes âgées de l'Ontario. Toronto: Imprimeur de la Reine pour I'Ontario.

Pelletier, J. et T. BOyer (1988). Méthode d'évaluation de l'implantation des services en santé mentale. Hull: Conseil régional de la santé et des services sociaux.

Pelletier, J. et T. BOYer (1988). "Programme ou services de maintien, de support, d'accompagnement, de réadaptation et de réinsertion dans la communauté, grille 7 », Méthode d'évaluation de l'implantation des services en santé mentale. Hull: Conseil régional de la santé et des services sociaux.

PRICE WATERHOUSE (1989). Étude opérationnelle du programme de soins à domicile de l'Ontario: rapport final. Toronto : ministère de la Santé de I'Ontario.

PRICE WATERHOUSE (1989). Évaluation des services intégrés d'aides familiales: rapport final. Toronto: ministère des Services sociaux et communautaires, Direction des services aux personnes âgées.

Sudbury (1991). Overview of the Seniors' Perception Study. Sudbury: Task Force for Seniors Advisory Council.

The Social Service ReSEARCH AND Advisory Group (SSRAG) (1992). Resources Directory. Sudbury: Human Services within the Districts of Sudbury and Manitoulin. 
An nexe I

\section{Programmes et services de soins de longue durée dans la Municipalité régionale de Sudbury}

\begin{tabular}{|c|c|c|c|}
\hline AGENCES & GESTION & FINANCEMENT & SERVICES \\
\hline Meals on Wheels & $\begin{array}{l}\text { Organisme bénévole } \\
\text { à but non lucratif }\end{array}$ & $\begin{array}{l}\text { Min. des Services } \\
\text { sociaux et communau- } \\
\text { taires, dons et contri- } \\
\text { butions des usagers }\end{array}$ & Popote roulante \\
\hline Finish Rest Home & $\begin{array}{l}\text { Organisme bénévole } \\
\text { à but non lucratif }\end{array}$ & $\begin{array}{l}\text { Contributions } \\
\text { des utilisateurs, min. } \\
\text { des Services sociaux } \\
\text { et communautaires } \\
\text { et du Logement }\end{array}$ & $\begin{array}{l}\text { Logements offrant } \\
\text { des services de soutien : } \\
\text { - vérification de sécurité } \\
\text { - repas communautaires } \\
\text { - transport } \\
\text { - entretien ménager } \\
\text { - visites d'amitié }\end{array}$ \\
\hline Service des malades & $\begin{array}{l}\text { Organisme bénévole } \\
\text { à but non lucratif }\end{array}$ & $\begin{array}{l}\text { Contributions } \\
\text { des membres }\end{array}$ & $\begin{array}{l}\text { Repas communautaires, } \\
\text { messe, activités sociales, } \\
\text { visite d'amitié }\end{array}$ \\
\hline Société d'Alzheimer & $\begin{array}{l}\text { Organisme à but } \\
\text { non lucratif }\end{array}$ & $\begin{array}{l}\text { Dons et min. des } \\
\text { Services sociaux } \\
\text { et communautaires }\end{array}$ & $\begin{array}{l}\text { Programme de jour, répit, } \\
\text { groupe de soutien } \\
\text { pour les familles }\end{array}$ \\
\hline $\begin{array}{l}\text { Organismes } \\
\text { de soins palliatifs }\end{array}$ & $\begin{array}{l}\text { Organisme bénévole } \\
\text { à but non lucratif }\end{array}$ & $\begin{array}{l}\text { Dons et contrat } \\
\text { de service avec le } \\
\text { Centre de cancérologie }\end{array}$ & $\begin{array}{l}\text { Entretien ménager, transport } \\
\text { et répit, visites d'amitié } \\
\text { et soutien à la famille }\end{array}$ \\
\hline Croix-Rouge & $\begin{array}{l}\text { Organisme à but } \\
\text { non lucratif }\end{array}$ & $\begin{array}{l}\text { Min. des Services } \\
\text { sociaux et communau- } \\
\text { taires et contributions } \\
\text { des usagers }\end{array}$ & $\begin{array}{l}\text { Prêts d'équipements } \\
\text { médicaux, entretien ménager, } \\
\text { visites d'amitié, transport et } \\
\text { programmes de prévention }\end{array}$ \\
\hline $\begin{array}{l}\text { Valley East et } \\
\text { Walden Home } \\
\text { Support Program }\end{array}$ & $\begin{array}{l}\text { Organisme à but } \\
\text { non lucratif }\end{array}$ & $\begin{array}{l}\text { Min. des Services } \\
\text { sociaux et } \\
\text { communautaires } \\
\text { et contributions } \\
\text { des usagers }\end{array}$ & $\begin{array}{l}\text { Entretien extérieur, entretien } \\
\text { ménager, transport, } \\
\text { réparations mineures } \\
\text { de la maison, peinture } \\
\text { et grand ménage }\end{array}$ \\
\hline $\begin{array}{l}\text { Infirmières de l'O rdre } \\
\text { de Victoria (VON) }\end{array}$ & $\begin{array}{l}\text { Organisme à but } \\
\text { non lucratif }\end{array}$ & $\begin{array}{l}\text { Dons, contrat avec } \\
\text { le Service de santé } \\
\text { publique et contri- } \\
\text { butions des usagers }\end{array}$ & $\begin{array}{l}\text { Coordination des placements, } \\
\text { soins infirmiers à domicile, } \\
\text { soins des pieds et programme } \\
\text { de jour pour personnes âgées }\end{array}$ \\
\hline
\end{tabular}


An nexe I (suite)

Programmes et services de soins de longue durée dans la Municipalité régionale de Sudbury

\begin{tabular}{|c|c|c|c|}
\hline AGENCES & GESTION & FINANCEMENT & SERVICES \\
\hline $\begin{array}{l}\text { Service de santé } \\
\text { publique } \\
\text { (soins à domicile) }\end{array}$ & $\begin{array}{l}\text { Organisme } \\
\text { paragouvernemental }\end{array}$ & $\begin{array}{l}\text { Min. de la Santé, } \\
\text { Municipalité régionale } \\
\text { de Sudbury }\end{array}$ & $\begin{array}{l}\text { Case management, } \\
\text { physiothérapie, ergothérapie, } \\
\text { orthophonie, services sociaux, } \\
\text { nutrition, pansements et } \\
\text { fournitures médicales, } \\
\text { prêts d'équipements médicaux } \\
\text { et aide à domicile } \\
\text { - entretien ménager } \\
\text { - préparation de repas } \\
\text { - aide à la toilette personnelle } \\
\text { - courses }\end{array}$ \\
\hline Manoir des Pionniers & Organisme municipal & $\begin{array}{l}\text { Municipalité régionale } \\
\text { de Sudbury, min. } \\
\text { des Services sociaux } \\
\text { et communautaires } \\
\text { et contributions } \\
\text { des usagers }\end{array}$ & $\begin{array}{l}\text { Hébergement, service } \\
\text { de relève, programme de jour } \\
\text { et soins infirmiers }\end{array}$ \\
\hline $\begin{array}{l}\text { Manoir de Garson } \\
\text { Extendicare York } \\
\text { Extendicare } \\
\text { Falconbridge }\end{array}$ & $\begin{array}{l}\text { Établissements privés } \\
\text { (maisons de soins } \\
\text { infirmiers) }\end{array}$ & $\begin{array}{l}\text { Contributions } \\
\text { des usagers } \\
\text { et min. de la Santé }\end{array}$ & $\begin{array}{l}\text { Hébergement et soins } \\
\text { infirmiers, lessive, épicerie, } \\
\text { etc. }\end{array}$ \\
\hline Résidence Regency & $\begin{array}{l}\text { Établissement privé } \\
\text { (maison de repos } \\
\text { et de retraite) }\end{array}$ & $\begin{array}{l}\text { Contribution } \\
\text { des usagers }\end{array}$ & Hébergement et loisirs \\
\hline Hôpital Laurentien & $\begin{array}{l}\text { Organisme } \\
\text { paragouvernemental }\end{array}$ & Min. de la Santé & $\begin{array}{l}\text { Hébergement pour soins } \\
\text { de longue durée, soins } \\
\text { gériatriques, évaluation } \\
\text { et réadaptation }\end{array}$ \\
\hline $\begin{array}{l}\text { Service social } \\
\text { municipal }\end{array}$ & Organisme municipal & $\begin{array}{l}\text { Municipalité régionale } \\
\text { de Sudbury }\end{array}$ & $\begin{array}{l}\text { Appareils et accessoires } \\
\text { médicaux }\end{array}$ \\
\hline
\end{tabular}




\section{AnNeXe II}

\section{Définition des concepts liés à l'accessibilité des services}

\section{Accessibilité}

Ce critère vise à évaluer la facilité et la commodité de l'accès à une variété de services, au moment opportun, selon les besoins des personnes qui demandent de l'aide et le plus près possible des milieux de vie ( $p$. 15.2)

\section{Accent sur la qualité de vie}

Ce critère vise à évaluer l'importance qu'accorde le programme à la promotion d'une qualité de vie des personnes qu'il aide, notamment le respect des droits et libertés, I'autonomie financière, le bien-être, la sécurité, l'estime de soi, la responsabilisation et la prise en main de leur situation (p. 15.15).

\section{Communautarisation}

Ce critère vise à évaluer jusqu'à quel point le programme, dans son approche, sa philosophie, son organisation et ses actions, se situe dans la communauté et la met à contribution (p. 15.13).

\section{Continuité}

Ce critère vise à évaluer l'importance accordée au cheminement à long terme des personnes (p. 15.8).

\section{Coordination}

Ce critère vise à évaluer les relations entre le programme et les autres programmes du secteur de la santé mentale* ou des secteurs connexes, la complémentarité de ses ressources et le synchronisme de ses interventions avec celles des autres programmes (p. 15.6).

\section{Personnalisation}

Ce critère vise à évaluer jusqu'à quel point les services, les ressources, les interventions, les supports sont pensés, administrés et actualisés à partir de la personne et de ses besoins (p. 15.10).

* Dans le contexte de cette recherche, il faut remplacer santé mentale par services à domicile.

Source: J. Pelletier et T. Boyer (1988). «Programme ou services de maintien, de support, d'accompagnement, de réadaptation et de réinsertion dans la communauté, grille 7 ", Méthode d'évaluation de l'implantation des services en santé mentale. Hull : Conseil régional de la santé et des services sociaux. 